\title{
Connectivity for the Frisbee Architecture
}

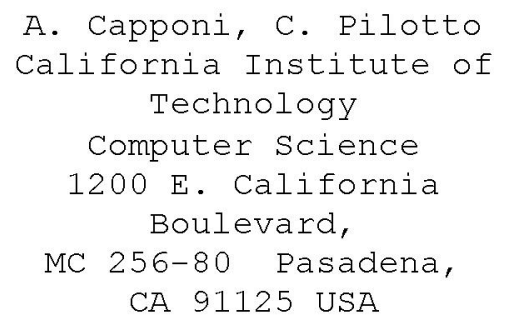

\author{
A. Farina, G.Golino \\ Selex SI \\ Via Tiburtina, $\mathrm{km} 12,400$ \\ 00131 Rome \\ Italy
}

afarina@selex-si.com ggolinodselex-si.com

\author{
L. Kaplan* \\ U.S. Army Research \\ Laboratory \\ 2800 Powder Mill Road, \\ Adelphi, MD 20783.
}

lkaplandieee.org

\begin{abstract}
In this paper we investigate the $k$ connectivity threshold of distributed dense ad hoc heterogeneous wireless sensor network architecture. We consider the situation when sensors are deployed in the surveillance area according to a uniform distribution perturbed by a Gaussian noise. We derive analytically the minimum detection range which guarantees an emerging structure in the network, namely the connectivity, which becomes larger and larger as the number of sensors in the network increase. This allows the target track to be propagated almost surely throughout the network using the minimum possible amount of prime energy. We report the results of some simulation experiments which further support the theoretical results.
\end{abstract}

Keywords : random distributed sensor networks, selforganization, connectivity threshold, detection range.

\section{Introduction}

Wireless sensor networks consist of large number of devices, each capable of some limited computation, communication and sensing, operating under energy constraints in an unattended mode [1]. These networks are intended for a broad range of environmental sensing applications from weather data-collection to target tracking and habitat monitoring [2], [3], [4].

In application such as battlefield surveillance and environmental monitoring, sensors may be dispersed, scattered, or airdropped in remote terrains.

*The views and conclusions contained in this document are those of the authors and should not be interpreted as presenting the official policies either express or implied of the Army Research Laboratory or the U. S. Government.
The sensors establish spontaneously a communication network, monitor the area in an energyefficient manner, and re-organize upon failure [5]. The sensors create a globally coherent pattern out of local interactions, i.e. they form a self-organizing system [6], [7]. Other examples of self-organizing system are flocks of birds, shoals of fish, swarms of bees in nature and self-organizing neural networks, swarm intelligence and self-configuring and adaptive sensor networks in engineered systems.

In order to exploit the full communication power of the network, it is important that any node can exchange information with the others. In this paper we assume that two nodes in the network can exchange information only if each of them falls within the coverage area of the other. Therefore, it becomes important to design the detection ranges of the sensors in such a way to guarantee that the network be connected with high probability. This means that information sent by a source node in the network can be propagated to a destination node through a communication path, i.e. through a sequence of intermediate nodes in the network. The smallest the length of the communication path, the smallest the propagation delay in the network.

Whatever is the sensor deployment strategy used (random, regular, planned deployment) there are always inherent uncertainties in the final sensor location. Generally, it is either impossible or very difficult to place sensors at the desired location; for instance, it may be impractible to disseminate sensors from an airplane onto a foreign territory for surveillance purposes under severe weather condition [8]. Furthermore, sensors cannot be expected to fall exactly at predetermined locations; rather there are regions where there is a high probability of a sensor being actually located. In underwater deployment sensors may move due to drift or water current. In hilly terrain sensors they may move due to slope of the terrain.

The primary contribution of this paper is to provide the design parameters of the wireless sensor network prototype proposed in the companion paper [9] in such way to enable an energy efficient target tracking and 
target detection. In particular we give the minimum detection range of complex sensors which gives almost surely the connectivity of the random sensor network. Section 2 recalls the layered architecture used, Section 3 formulates the problem using the theory of geometric random graphs. Section 4 describes how the connectivity of the sensor network relates to the propagation of the track estimate throughout the network. Section 5 presents experimental results and comparison with the theory. Section 6 summarizes the main findings.

\section{The sensor network architecture}

Sensors are spread out over a two dimensional and squared surveillance area. Initially the locations are randomly chosen according to a uniform distribution. However, when sensors are dropped on the surveillance area, the final location may not correspond with the one initially chosen; we model this uncertainty using a bivariate Gaussian distribution centered at the intended sensor location.

Our network consists of two types of sensors, simple and complex. As in [10], simple sensors have only the capability of sensing their coverage area, comp ute binary information and transmit data to complex sensors. Binary information is encoded by a 1 if sensor detects something crossing its coverage area and by a 0 otherwise. Complex sensors, instead, have computation capabilities; they are able to locate the target by applying the maximum likelihood estimation algorithm described in [10].

Sensors exchange information only if they are close, i.e. if the overlapping of their coverage areas is greater than a fixed constant (strong overlapping). Simple sensors communicate with complex sensors and complex sensors with both simple and complex ones. A clustering architecture has been implemented: simple sensors are the elements of the clusters and complex ones are the heads of the clusters.

Our objective is to give the design parameters of the sensors in the network which minimize the costs while guaranteeing the connectivity of the sensor network consisting of only complex sensors.

When the target track estimation is carried out, only a small subset of sensors in the network is kept in its fully active state. The details of how these sensors are selected are dis cussed in the companion paper [9]. We call such a subset of the sensor network the active zone. The active zone moves through the network along with the target. The transmission protocol consists of three stages:

- Each complex sensor awakes the simple sensors in its cluster.

- Simple sensors monitor their coverage area, and when they detect the presence of a target, awake close complex sensors, transmit their binary information to them and fall asleep
- Complex sensors collect data from simple sensors, process their information as in [10] and compute the estimated position of the target. Finally they choose which complex sensors will be used to detect the target at the next step and activate a mechanism to awake them.

The third stage of the protocol guarantees that the estimate is propagated throughout the network; however if the network consisting of complex sensors is not connected, there is a risk that all the sensors in the network will become asleep and consequently the target track estimation gets lost and is never recovered. This is an undesirable feature which we want to avoid. To this purpose we:

1) Calculate the minimum transmitting range which guarantees the network consisting of only complex sensors to be $k$-connected almost surely (Section 3).

2) Outline an algorithm which propagates the target track estimation to the new active zone efficiently (Section 4).

Item 1) allows minimizing the amount of energy used, while item 2) implements the propagation of the track estimate through the network (which is connected almost surely).

\section{The connectivity problem}

\subsection{Preliminaries}

A random graph $G(n, p(n))$ is a graph on $n$ nodes such that edges are selected independently with probability $p(n)$ [11]. Such a model is not well suited for our problem since edges depend on the geometric distance between the nodes and consequently are correlated random variables.

Our problem may instead be modeled by means of geometric random graphs [12]. In this graph each node is uniquely identified by its $x, y$-coordinates which are selected according to some probability distribution and an edge between two nodes occurs if and only if their distance is smaller than a certain threshold valuer $r$.In our case the nodes of the graph are the complex sensors and sensor $x$ and $y$ can only communicate if there exists an edge connecting them. The detection range of the sensor is set to be the threshold value $r$.

Therefore, we are simply saying that an edge is inserted between two sensors if and only if each of them falls within the coverage area of the other. Thus we have stated that an overlapping between two sensors is strong if and only if each of the two sensors falls within the coverage area of the other sensor. 
A graph $G$ is connected if for any pair of nodes $x$ and $y$, it is possible to go from $x$ to $y$ traversing the edges and nodes of the graph. A generalization of connectivity to $k$-connectivity is the following: a graph $G$ is said to be $k$-connected if it cannot be disconnected by the removal of $k-1$ or fewer vertices. Clearly, 1connectivity corresponds to connectivity.

Our objective is the following: what is the minimum value of the detection range $r(n)$ which guarantees that the sensor network is $k$-connected with probability one as the number of sensors $n$ goes to infinity?

We call such a value the $k$-connectivity threshold for the $\operatorname{graph} G$ and denote it by $r_{k}$.

The degree of a node $v$ in a graph is defined to be the number of nodes which are connected to $v$ by an edge. The minimum degree of a graph is the minimum degree over all its nodes. Denote by $\delta_{k}$ the threshold value of $r$ above which a random geometric graph $G$ has minimum degree at least $k$ with probability one. Penrose [12] proves the following theorem.

Theorem 1. Given a positive integer $k$ and a geometric random graph $G$, for any probability distribution on the nodes we have

$$
r_{k}=\delta_{k}
$$

with probability one as the number of nodes in the graph goes to infinity

One of the first results concerning the connectivity of wireless networks was published in [13]. The study of the connectivity problem in two dimensions was then reconsidered in [14] and [15]. In [16] and [17], using recent results from the theory of geometric random graphs [18], the connectivity threshold function for a uniformly distributed ad hoc network in $[0,1]^{2}$ is obtained.

In the mathematical literature, Apple and Russo [19] investigate the special case when points are uniformly distributed in the unit square and the metric is taken to be the $l_{\infty}$ norm. Penrose [18] generalizes their connectivity results to any metric $l_{p}, 2 \leq p \leq \infty$, proving that asymptotically, with high probability, if one starts with single vertices and adds the corresponding edges as the radius increases, the resulting graph becomes $(k+1)$ connected at the moment it achieves a minimum degree of $(k+1)$.

The reason why we are interested in the minimum is that sensors with small detection range are usually cheaper and all this allows for the possibility of more sensors to be deployed.

\subsection{Setup and solution of the problem}

We assume the coordinates representing sensor positions to be initially chosen according to a probability distribution $p$ uniform on the square $\Omega=[0,1]^{2}$. Due to uncertainty factors (see Section 1) we assume that each sensor falls within a certain ellipsoid centered at the aimed sensor location.

We want to establish a reliable and fault tolerant communication infrastructure in that square. The fault tolerant property of the network depends on the $k$ connectivity. The larger $k$, the more the network can be considered fault tolerant. However, if the value of $k$ is too large, then a larger detection range is needed and therefore the sensors must be more costly

The marginal probability density functions pdf on the nodes are:

$f_{X}(x)=\int_{0}^{1} \frac{1}{\sqrt{2 \pi} \sigma} e^{-\frac{(x-z)^{2}}{2 \sigma^{2}}} d z$

and

$f_{Y}(y)=\int_{0}^{1} \frac{1}{\sqrt{2 \pi} \sigma} e^{-\frac{(y-z)^{2}}{2 \sigma^{2}}} d z$

which may also be rewritten as

$f_{X}(x)=\frac{1}{2}\left(-\operatorname{erf}\left(\frac{-1+x}{\sqrt{2} \sigma}\right)+\operatorname{erf}\left(\frac{x}{\sqrt{2} \sigma}\right)\right)$

and

$f_{Y}(y)=\frac{1}{2}\left(-\operatorname{erf}\left(\frac{-1+y}{\sqrt{2} \sigma}\right)+\operatorname{erf}\left(\frac{y}{\sqrt{2} \sigma}\right)\right)$

where erf () denotes the error function.

Since we are interested in the pdf only inside the unit square, we consider the expressions (4) and (5) which are defined on the whole real axis and normalize them in the unit square. In order words, we define the new marginal $f_{X}^{*}(x)$ and $f_{Y}^{*}(y)$ as

$$
f_{X}^{*}(x)= \begin{cases}\frac{f_{X}(x)}{\sqrt{c_{\sigma}}}, & 0 \leq x \leq 1 \\ 0 & \text { otherwise }\end{cases}
$$

and

$f_{Y}^{*}(y)= \begin{cases}\frac{f_{Y}(y)}{\sqrt{c_{\sigma}}}, & 0 \leq y \leq 1 \\ 0 & \text { otherwise }\end{cases}$

where $\int_{0}^{1} f_{X}(x) d x \int_{0}^{1} f_{Y}(y) d y$ which is equal to

$c_{\sigma}=\sigma^{2}\left(\left(-1+e^{-\frac{1}{2 \sigma^{2}}}\right) \sqrt{\frac{2}{\pi}}+\frac{\operatorname{erf}\left(\frac{1}{\sqrt{2} \sigma}\right)}{\sigma}\right)^{2}$ 
The $k$-connectivity connectivity threshold for the random geometric graph with the above derived probability distribution $f^{*}$ is derived in Appendix 1 . Here, only the result is stated:

$r_{\alpha}=\sqrt{\frac{\log (n)}{n \pi f_{\text {min }}^{*}}}$

We notice that the connectivity threshold $r_{k}$ is independent of the particular $k$ as it appears from formula (9). However, when the number of sensors $n$ is fixed, it is reasonable to expect that the $k$-connectivity threshold becomes a decreasing function of $k$.

\section{Routing Algorithm}

In section 3 we have derived the minimum detection range which guarantees with probability one the $k$ connectivity of the network of complex sensors. While this is a necessary condition for the estimate to be propagated, it is not sufficient. What can happen is that two consecutive active zones are connected to each other by another sensor which is in neither of the two active zones (see Figure 1). If a complex sensor only transmits its track estimate to his adjacent sensors in the graph, we would have that the nodes in the active zone at step $t+1$ would not be awaken by the nodes in the active zone at the previous step $t$ and therefore would not receive their estimate. Therefore the target is lost and never recovered because the entire network is shut down from this moment on.

In order to assure that the track estimate is propagated to the next active zone with large probability we can use a routing algorithm such as a flooding with pruning algorithm. Each complex sensor in the current active zone broadcast a packet containing the track estimate to all adjacent complex sensors. Upon receiving the packet each sensor transmits the packet to all its adjacent sensors except to the one from which the packet was received. In order to reduce the number of packets sent and therefore the amount of energy wasted for propagating the information throughout the network, the complex sensor which sends the first packet may insert a sequence number into it. For example, such sequence number may be set to the diameter of the network, i.e. to the maximum distance between any pair of nodes in the network. Each receiving sensor decrements the sequence number before sending the packet further along the network and does not send the packet if the value of the sequence number becomes zero.

\section{Simulation Results}

In all simulations we have fixed the standard deviation of the bivariate normal to be 0.02 along each component.
Since the problem of deciding whether a graph is $k$ connected is NP-complete and would require us a considerable amount of time, in our numerical simulations we have estimated the probability of $k$ connectivity with the probability that the minimum degree of the graph is $k$. Our choice of using the minimum degree can be justified by Theorem 1 .

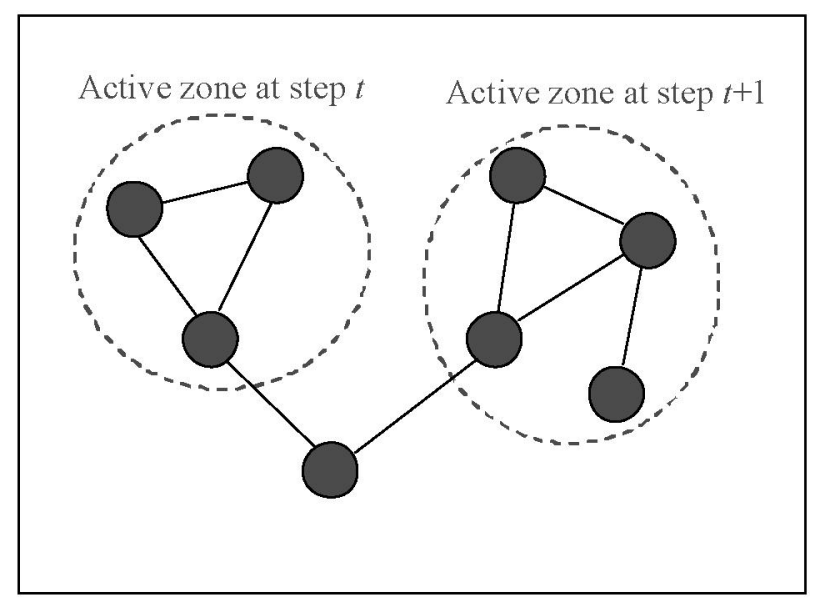

Figure 1.

\section{Active zones at two subsequent time steps.}

The first experiment shows how the probability of 1connectivity varies as a function of the sensor detection range. Such probability has been estimated by means of Monte Carlo runs. As expected, Figure 2 shows that the probability of 1-connectivity increases faster for large number of sensors $(\mathrm{N}=200)$. For smaller number of sensors, e.g. $\mathrm{N}=20$, the probability of 1-connectivity starts approaching one only for values of the detection range equal to about 0.5 , i.e. about half of the side of the squared surveillance area.

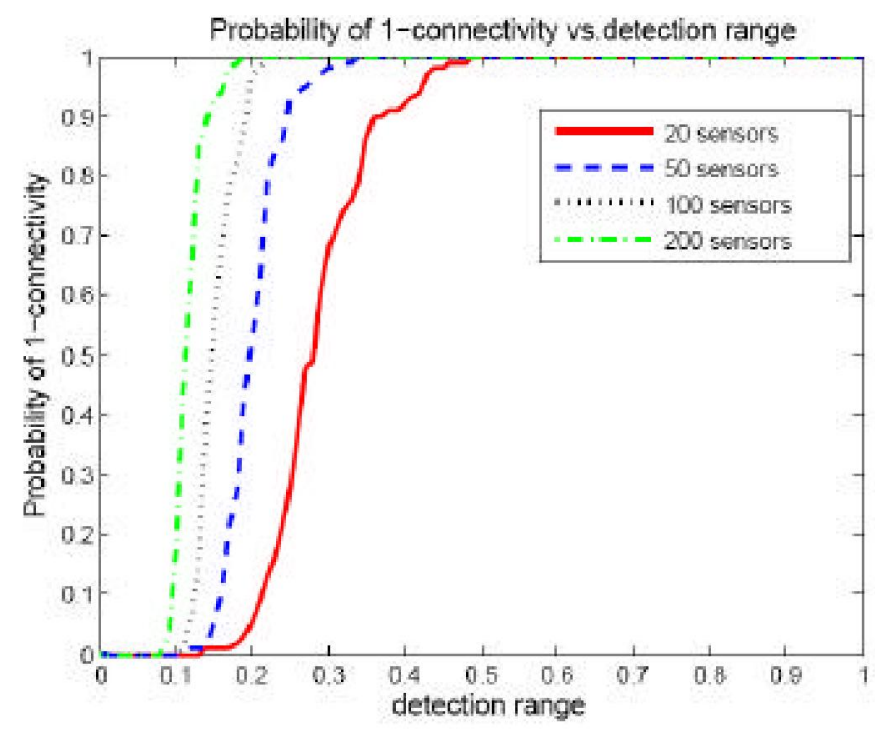




\section{Figure 2.}

Probability of 1 -connectivity versus detection range for different number of sensors in the network

The following table shows for the different sensor configurations the detection range for which the probability of connectivity in Figure 2 is greater than 0.9 .

\begin{tabular}{|c|c|}
\hline Number of Sensors & Detection Ranges \\
\hline 20 & 0.4297 \\
\hline 50 & 0.3106 \\
\hline 100 & 0.2383 \\
\hline 200 & 0.1807 \\
\hline
\end{tabular}

Table 1.

Detection range for different sensor configurations.

The second experiment presents some simulatic results concerning the probability of $k$-connectivity of sensor network whose sensors are deployed accordin to $f^{*}$. The number $n$ of sensors is fixed to thre thousand

The detection range of each sensor equals the $k$ connectivity threshold, which is independent of $k$. Th $k$-connectivity threshold for the distribution $f^{*}$ calculated using the formula given in equation (9). Th obtained results are summarized in Figure 3 for a numb of three thousand sensors.

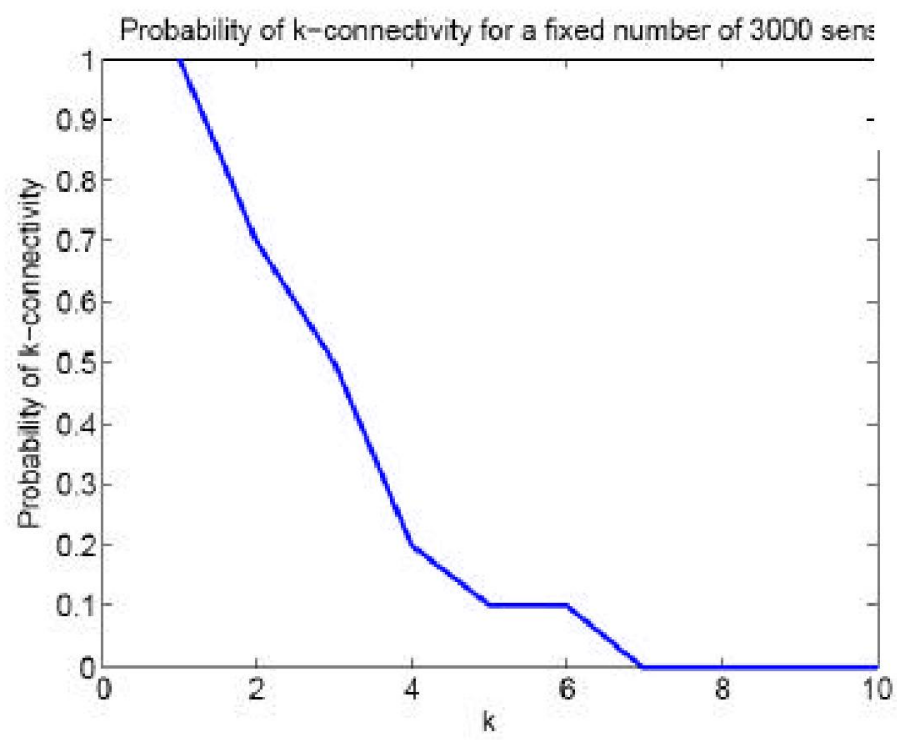

Figure 3.

Probability of $k$-connectivity for a network of three-thousand sensors using the connectivity threshold
The final experiments shows how the detection range computed using (9) compares with the expected value of the minimum detection range which guarantees 1connectivity. The latter is estimated as follows: for each given number of sensors in the network we run onehundred Monte Carlo runs. For each run we keep track of the minimum value of the detection range for which the graph generated by the run is 1-connected. Finally, we average the values computed earlier.

The number of sensors in the network varies from ten to one thousand. Figure 4 shows that the two detection ranges are very close to each other, especially when the number of sensors becomes larger than two-hundred Therefore, the theoretical predictions gives by (9) starts becoming effective for a number of sensors on the order of few hundreds and thus not require the number of sensors in the network to be extremely large.

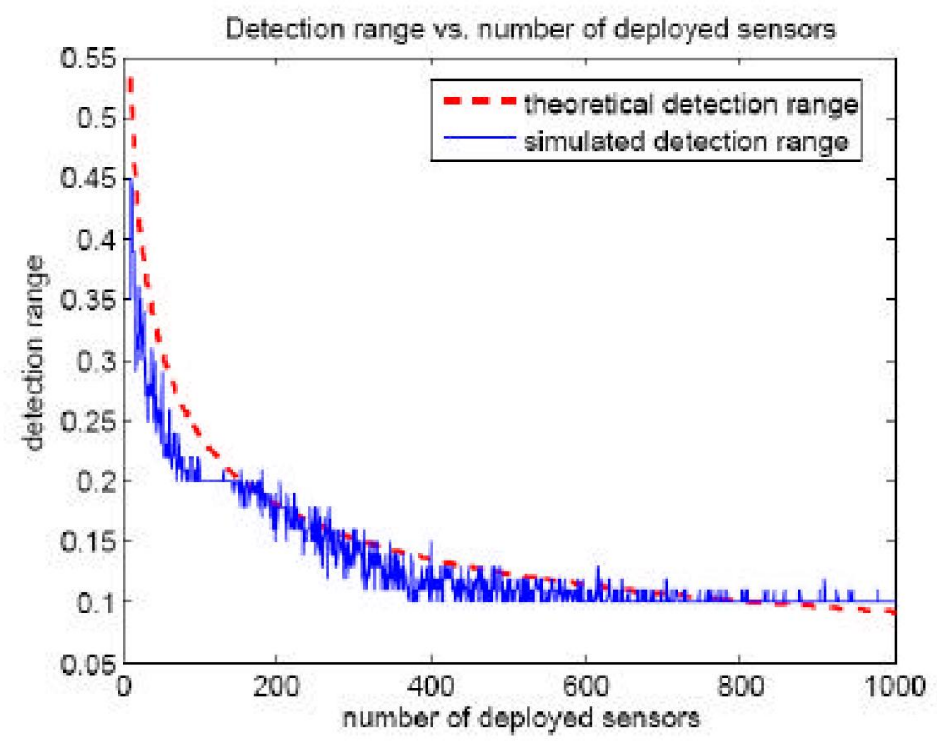

Figure 4.

Connectivity threshold versus number of deployed sensors.

\section{Conclusions}

In this paper we have investigated the minimum detection range required to guarantee the $k$ connectivity of our proposed distributed sensor network architecture. We have assumed that sensors are initially deployed uniformly, and then perturbed by a Gaussian noise. Maintaining the $k$-connectivity for the network is essential if we want a fault-tolerant network where the target track estimate is propagated with probability one along the network without risking that the sensor network shuts down and do not recover any more the target. However, the condition is not sufficient and must be supported by efficient flooding algorithms which 
distribute the estimation to the nodes of the network in proximity of the target. The results of this paper have been exploited in the companion paper [9].

In a future continuation of the work we would like to consider a random sensor network of simple sensors and allow them to sleep and wake-up via some random protocol. We would then like to demonstrate how the expected number of awaken nodes at some time instant can be used to predict both the connectivity of the network and the localization performance of the network estimated via the root mean squared position error of the simple sensors.

\section{References}

[1] F. Zhao and L. Guibas. "Wireless sensor networks: an information processing approach". Morgan Kaufmann Publishers, 2004.

[2] A. Farina, G. Golino, A. Capponi, C. Pilotto. "Surveillance of wide areas by means of a random sensor network: a heterogeneous sensor approach". Proceedings of the $8^{\text {th }}$ International Conference on Information Fusion, FUSION05, Philadelphia, USA, 2005.

[3] A. Cerpa, J. Elson, D. Estrin, L. Girod, M. Hamilton and J. Zhao. "Habitat monitoring: application driver for wireless communications technology". Proceedings of the 2001 ACM SIGCOMM Workshop on Data Communications in Latin America and the Caribbean, San Jose, Costa Rica, pp. 20-41, 2001.

[4] A. Mainwaring, J. Polastre, R. Szewczyk, D. Culler and J. Anderson. "Wireless sensor networks for habitat monitoring". Proceedings of the $\mathrm{p}^{\mathrm{t}} \mathrm{ACM}$ International Workshop on Wireless Sensor Networks and Application, WSNA02, Atlanta, USA, pp. 88-97, 2002.

[5] D. Estrin, R. Govindan, J. Heidemann and S. Kumar. "Next century challenges: scalable coordination in sensor networks". Proceeding of the $5^{\text {th }}$ annual $\mathrm{ACM} / \mathrm{IEEE}$ International conference on Mobile Computing and Networking, MOBICOM, Seattle, USA, pp. 263-270, 1999

[6] F. Heylighen, "The Science of self-organization and adaptively", Encyclopaedia of Life Support Systems (EOLSS Publishers Co. Ltd), 2001.

[7] T. C. Collier, C. Taylor, "Self-organization in sensor networks", Journal of Parallel and Distributed Computing, in press.

[8] P. Corke, S. Hrabar, R. Peterson, D. Rus, S. Saripaui and G. Sukhatme. "Autonomous deployment and repair of sensor network using an unmanned aerial vehicle". IEEE International Conference on Robotics and Automation (ICRA), New Orleans, USA, Vol. 4, pp. 3602-3608, 2004

[9] A. Capponi, A. Farina, G. Golino, L. Kaplan, C. Pilotto, "Algorithms for the selection of the active sensors in distributed tracking: comparison between the Frisbee and the GNS methods". Proceedings of $9^{\text {th }}$ International Conference on Information Fusion, Florence, Italy, 2006.

[10] M. Rodriguez, M. Lazaro, and L. Tong. "Target location estimation in sensor networks using range information". IEEE Sensor Array and Multichannel Signal Processing workshop, 19-21, July 2004, Sitges, Spain.

[11] B. Bollobás. Random graphs. Academic Press, 1985.

[12] M.D. Penrose. Random geometric graphs. Oxford studies in probability 5 Oxford University Press, 2003.

[13] P. Gupta and P.R. Kumar. "The capacity of wireless networks". IEEE Transaction on Information Theory, vol. 46 , no. 2, pp. 388-404, 2000.

[14] Y.-C. Cheng and T. Robertazzi. "Critical connectivity phenomena in multihop radio models". IEEE Transaction on Communication, vol. 37 , no. 7, pp. 770-777, 1989.

[15] P. Gupta and P.R. Kumar. "Critical power for asymptotic connectivity in wireless networks". In Stochastic Analysis, Control, Optimization and Applications: A Volume in Honor of W. H. Fleming, W.M. McEneany, G. Yin and Q. Zhang, Eds. Boston, MA, USA, pp. 547-566, 1998.

[16] P. Panchapakesan and D. Manjunath. "On the transmission range in dense ad hoc radio networks". In Proceedings of Conference on Signal Processing and Communications, SPCOM01, Bangalore, India, Paper 01-38, 2001.

[17] C. Bettstetter. "On the minimum need degree and connectivity of a wireless multihop network". Proceedings of the $3^{\text {rd }}$ ACM International Symposium on Mobile Ad Hoc Networking and Computing, MobiHoc, Lausanne, Switzerland, pp. $80-91,2002$.

[18] M.D. Penrose. "On $k$-connectivity for a geometric random graph". Random Structures and Algorithms, vol. 15, no. 2, pp. 145-164, 1999.

[19] M. Appel and R. Russo. "The connectivity of a graph on uniform points on $[0,1]^{d "}$. Statistics and Probability Letters, vol. 60, no. 4, pp. 351-357, 2002.

\section{Appendix}

Consider a two-dimensional area $\Omega=\left[0, l_{1}\right] \times\left[0, l_{2}\right]$. For $1 \leq j \leq 2$ denote by $\partial_{j}$ the union of all intersections of $j(d-1)$-dimensional hyperplanes bounding $\Omega$. Let $f_{j}^{*}$ be the infimum of $f^{*}$ over $\partial_{j}$. Furthermore, denote by $f_{0}{ }^{*}$ the infimum of $f^{*}$ over $\Omega$ and assume $f^{*}$ to be strictly positive on $\Omega$. Set 
$H(a)=1-a+a \log (a)$ for positive $a$ and 1 for $a=0$.

Then the following holds:

Theorem 2 [19]. Let $\left\{k_{n}\right\}_{n \geq 1}$ be a sequence of nonnegative integers such that $\lim _{n \rightarrow \infty} k_{n} / n=0$ and $\lim _{n \rightarrow \infty} k_{n} / \log (n)=b, \quad$ for $b \in[0, \infty)$. Furthermore, assume that the sequence $\left\{k_{n}\right\}_{n \geq 1}$ is non-decreasing, and define $a_{0}, a_{1} \in[0,1)$ by

$\frac{a_{j}}{H\left(a_{j}\right)}=\frac{2 b}{2-j}$

Then with probability 1 ,

$$
\lim _{n \rightarrow \infty} \frac{n \pi\left(r_{\kappa_{n}+1}\right)^{2}}{\log (n)}=\max \left(\max _{0 \leq j \leq 1}\left(\frac{2^{j}(2-j)}{2 f_{j}^{*} H\left(a_{j}\right)}\right), \frac{4 b}{f_{2}^{*}}\right)
$$

Notice that our joint probability density function which is given by the product of the densities $f_{X}^{*}(x)$ and $f_{Y}^{*}(y)$ is strictly positive on its domain. Furthermore, $f_{X}^{*}(x)$ and $f_{Y}^{*}(y)$ are both continuous concave functions defined on a bounded domain and symmetric with respect to the point 0.5 .

Suppose we wish to achieve $k$-connectivity. Applying Theorem 2 to our problem, we obtain after straightforward calculation that

$$
\lim _{n \rightarrow \infty} \frac{n \pi\left(r_{\kappa_{n}+1}\right)^{2}}{\log (n)}=\max \left\{\frac{1}{f_{\min }^{*}, 0}\right\}
$$

Therefore,

$$
r_{k}=\sqrt{\frac{\log (n)}{n \pi f_{\min }^{*}}}
$$

where $f_{\min }^{*}$ is the minimum of the joint probability density on the considered domain. 